\title{
Understanding taste and texture perception to enhance vegetable acceptance
}

\author{
E.M. Duffy ${ }^{1}$, J.E. Hayes ${ }^{2}$ and E.L. Feeney ${ }^{1}$ \\ ${ }^{1} U C D$ Institute of Food and Health, University College Dublin, Belfield, Dublin 4, Ireland and \\ ${ }^{2}$ Department of Food Science, Pennsylvania State University, PA 16802, USA
}

Food selection and ingestion is affected by a multitude of sensory, economic, genetic, psychological and biological factors ${ }^{(1-3)}$. Of these, taste is paramount, and humans have an innate preference for sweetness and aversion for bitterness; these are linked to vegetable acceptance ${ }^{(2)}$. Sufficient vegetable intake offers many health benefits ${ }^{(4)}$, however many populations fail to meet recommended guidelines ${ }^{(4-6)}$. With the rise in diet-related disease ${ }^{(4)}$, there is a need to better understand barriers to liking and intake. Here, we investigated predictors of vegetable liking in an American population, with a focus on taste and textural characteristics.

Participants $(n=92)$ aged $18-40$ years took part in this laboratory-based study. They were served broccoli, carrots and kale prepared in three ways (boiled, blended, raw). 'Overall' and 'texture' liking for each sample was rated on a general Labelled Magnitude Scale. Other ratings for a range of taste and textural properties were also obtained, and vegetable intake was measured. Statistical analyses were conducted using SPSS v20. Liking and self-reported intake are typically correlated; here, we found acute intake (in grams) was also correlated in with liking (Fig. 1). Generally, more bitterness decreased vegetable liking, whilst more sweetness increased liking $(p \leq 0 \cdot 001)$. Fig. 2 depicts the mean sweetness and bitterness intensity for each vegetable group alongside its' mean overall liking score. Additionally, preparation method and vegetable type both had a significant influence on overall liking $(p<0.001)$ (Fig. 3). Multiple linear regression analysis showed that over $60 \%$ of the variation in overall liking scores could be attributed to the range of sensory taste and texture properties examined here $\left(R^{2} \operatorname{Adj}=0 \cdot 61, p \leq 0 \cdot 001\right)$.

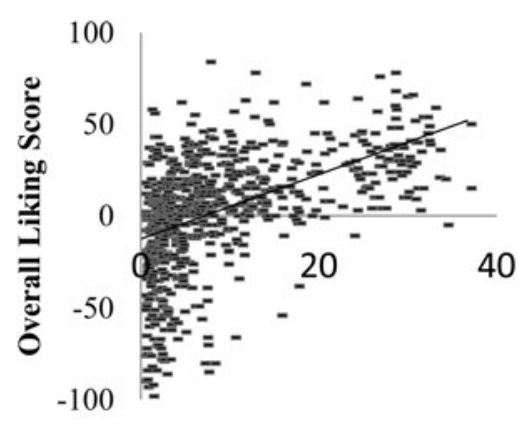

Intake (grams)

Fig. 1. Correlation between vegetable intake and linking.

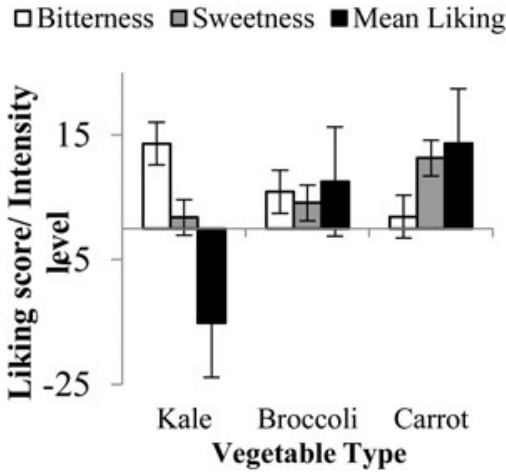

Fig. 2. Mean bitterness, sweetness overall linking for each vegetable.

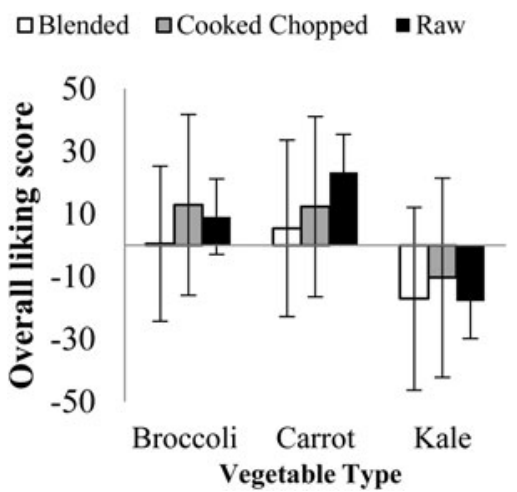

Fig. 3. The mean overall linking score for three vegetables.

Eating behaviours are complex, and vegetables intake is influenced by many factors. However, the present analyses demonstrate that taste and texture characteristics may explain considerable variation in vegetable liking, in a controlled setting. This information can be used to inform the design of future interventions, to actively increase vegetable consumption by providing access to vegetables that are prepared in a sensorially appealing manner, in order to promote liking and intake.

1. Nestle M, Wing R, Birch L, DiSogra L, Drewnowski A, Middleton S, et al. (1998) Behavioral and social influences on food choice. Nutrition reviews. 56(5), 50-64.

2. Dinehart ME, Hayes JE, Bartoshuk LM, Lanier SL, Duffy VB. (2006) Bitter taste markers explain variability in vegetable sweetness, bitterness, and intake. Physiology \& Behavior. 87(2), 304-313.

3. Drewnowski A. (1997) Taste preferences and food intake. Annual review of nutrition. 17, 237-253.

4. WHO. (2003) Diet, nutrition, and the prevention of chronic diseases: report of a joint WHO/FAO expert consultation: Diamond Pocket Books (P) Ltd.

5. McGuire S. (2013) State indicator report on fruits and vegetables, 2013, Centers for Disease Control and Prevention, Atlanta, GA. Advances in Nutrition: An International Review Journal. 4(6), 665-666.

6. EUFIC. EUFIC Review: Fruit and vegetable consumption in Europe - do Europeans get enough? 2012. 